\title{
Optical tunneling effect of surface plasmon polaritons and localized surface plasmon resonance
}

\author{
Wei-Chih Liu* \\ Department of Physics, National Taiwan Normal University, Taipei, Taiwan 11650, Republic of China \\ Din Ping Tsai \\ Department of Physics, National Taiwan University, Taipei, Taiwan 10617, Republic of China
}

(Received 4 September 2001; published 4 April 2002)

\begin{abstract}
Surface plasmon resonance of periodic narrow-grooved metallic thin films were studied with finitedifference time-domain simulations. Two distinguishable modes, surface plasmon polaritons and localized surface plasmons, are associated with different periods. The surface plasmon polaritons have widely extended near-field distributions and sharp resonance; however, the localized surface plasmon modes are highly concentrated in the grooves, and have broad resonance. Our results demonstrate that the surface plasmon polaritons and localized surface plasmons were coupled with electromagnetic waves to resonantly tunnel through the array of subwavelength holes. This optical tunneling effect exhibited large phase lag and time delay for the surface plasmon polariton. The broadness of localized surface plasmon resonance indicated that it could exist in randomly distributed, narrow-grooved structures as well.
\end{abstract}

DOI: 10.1103/PhysRevB.65.155423 PACS number(s): 78.66.Bz, 42.79.Dj, 42.82.Et, 71.36.+c

\section{INTRODUCTION}

A flat metallic surface or thin film is almost a perfect reflector for visible light. For example, a flat silver film with thickness $t$ larger than several ten nanometers is effectively opaque. An imposed surface structure may, however, significantly alter the optical response of a metal surface. It is well known that a periodic structure, like a grating, can reflect incident light into diffracted orders. A periodic metallic structure can also provide the in-plane momentum required for the incident light in the appropriate polarization to excite a surface plasmon polariton (SPP), resulting in strong optical absorption. This effect has been extensively studied in the last century. ${ }^{1,2}$

A closely related development is the study of extraordinarily high optical transmission through metallic films with a periodic array of subwavelength holes by Ebbesen and co-workers ${ }^{3-6}$ At resonant frequencies, the enhancement on transmission can be several orders of magnitude compared to what is predicted for a subwavelength aperture. ${ }^{7}$ The locations of transmission peaks correspond to the SPP resonance modes. Consequently, the phenomenon was explained as plasmon-enhanced light tunneling through subwavelength holes. $^{8-11}$

On the other aspect, nanoscale noble-metal structures can exhibit anomalous optical excitation in the visible range due to the localized surface plasmon (LSP). The LSP resonance positions depend mainly on the structure geometry and the polarization state of the incident light and the LSP excitation is accompanied with a highly localized field enhancement around the nanostructures. With fast progress of near-field optics and nanolithography technology, these properties make such metallic nanoscale structures especially interesting in the future micro- or nanophotonic applications.

The investigation of the LSP resonance on zeroth-order metal gratings, which are gratings with periods smaller than the wavelength of incident light, also intended to shed light on the physical mechanism of resonant tunneling of light through thin metal films. LSP resonant excitation on zeroorder gratings by incident TM-polarized radiation ( $\mathrm{H}$ field parallel to the grating grooves) results in strong field enhancement and absorption. Several researchers studied theoretically a metallic grating with very narrow slits, ${ }^{12-15}$ and found that TM-polarized incident light can excite LSP modes in the slits, and lead to large transmission through the grating. However, there is a major difference between a film with an array of subwavelength holes and a film with subwavelength slits, which is that only a subwavelength slit can support propagating modes traveling along the direction perpendicular to the film plane. Therefore Tan et al. proposed to use a silver film with narrow-grooved zeroth-order grating on both sides to overcome this problem. ${ }^{16}$ They found that LSP modes localized in the grooves of the two opposite surfaces, and led to high transmission in the visible and ultraviolet regions for TM-polarized incident light.

In this paper we chose a model similar to the work of Tan et al. to show that there are two different mechanisms for enhanced optical transmission through thin metal films with periodic structures at different periods. To reduce the complexity resulted from the metallic dispersion at different incident frequencies, we fixed the wavelength of the incident light at $650 \mathrm{~nm}$ and changed the periods instead. Though the resonant modes of a one-dimensional periodic system are not the same as those of a two-dimensional periodic system, it is still important to study the special characteristics of SPP and LSP resonance modes. They appear in much broader areas than the subwavelength hole arrays, and are useful tools to control, and selectively transport light.

\section{NUMERICAL MODEL}

The model structure is a free-standing thin silver film with periodic Gaussian-shaped grooves on both surfaces. As in Fig. 1, the profiles of the left and the right surfaces are symmetric. The depth of these grooves, $d$, is less than $t / 2$, where $t$ is the maximum thickness of the film. The minimum sepa- 


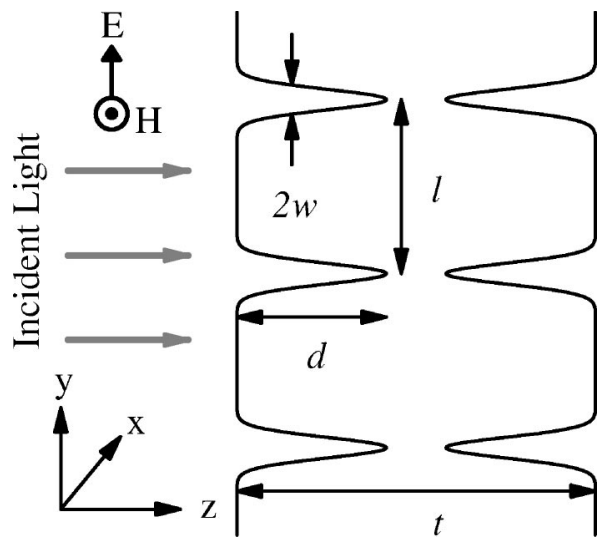

FIG. 1. A schematic illustration of the thin-film grating.

ration between the grooves on the opposite surfaces is larger than zero; therefore there should be no propagating electromagnetic modes between the left and the right surfaces, that is a situation rather more similar to the hole experiment of Ebbesen et al. than the narrow slits model. In our calculation, we assume that all the silver films have the same groove profile, the depth of the grooves, $d=50 \mathrm{~nm}$, and the width of the grooves, $w=10 \mathrm{~nm}$, while the film thickness $t$ is varied from 104 to $116 \mathrm{~nm}$. The period $l$ between the adjacent grooves is always less than the wavelength of incident light, $\lambda=650 \mathrm{~nm}$. The normally incident TM-polarized light the $\mathrm{H}$ field is parallel to the grooves, i.e., the direction of the incident field polarization is along the $y$ direction in Fig. 1) is from the left side of the silver film. In previous research, the period of the periodic structure was fixed and the optical responses for different frequencies of the incident light were studied. Because the noble metals are highly dispersive in the visible and ultraviolet regions, the fixed-period approach encountered varied indices of refraction of the noble-metal film at different frequencies, and substantially confounded the results. In this work, we fixed the frequency (and wavelength) of the incident light, and changed the periods of the structures to eliminate the complicated effects of dispersive properties. The control variables of the SPP and LSP resonance is the period only, that is the in-plane momentum provided by the periodic structure.

The numerical method used in this work is based on the two-dimensional finite-difference time-domain (FDTD) method with the perfectly matched layer (PML), ${ }^{17}$ because of its computational advantages of reduced memory requirements, freedom from matrix inversion issues, and ease in treating complex materials and shapes. It allows the calculations of both reflection and transmission of systems, and near-field distributions. To implement the FDTD approach, a region of space is discretized into a mesh of square cells. Maxwell's equations in differential form are solved directly. Material parameters are attributed to each cell of the mesh to create a discrete grid representation of the silver film, and the second-order Lorentz dispersion model ${ }^{18}$ is used to simulate the metallic materials. To minimize the size of the required grid, the perfect matched layer boundary conditions ${ }^{19,20}$ are applied to the left and right boundaries of the region shown in Fig. 1. In this work, the index of refraction of silver is
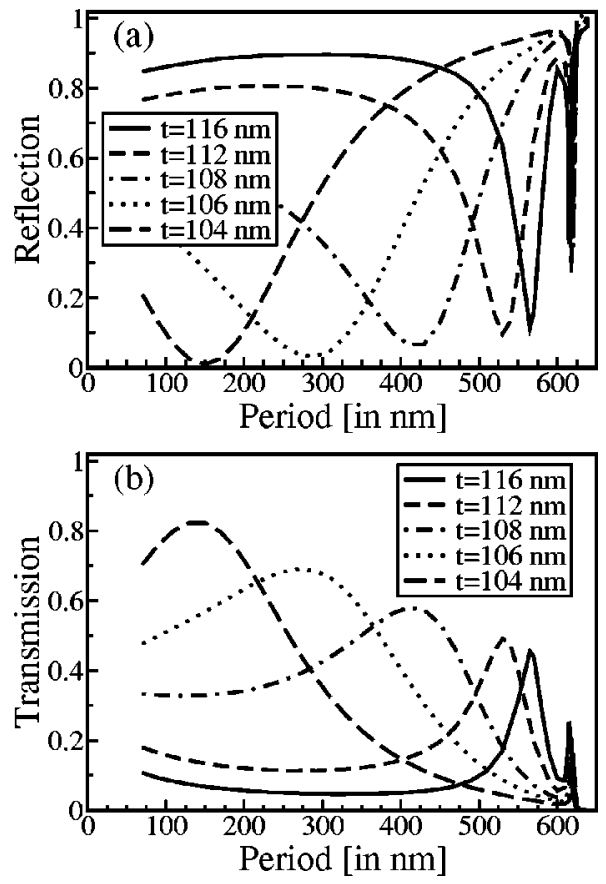

FIG. 2. (a) Reflection and (b) transmittance coefficients as functions of the period. The thickness of the films are from 104 to 116 nm.

$0.0555-4.44 i$ when the wavelength of incident light is 650 $\mathrm{nm}$. The silver film is surrounded by air, which has a frequency-independent index of refraction $n=1.0$. Our calculations were carried out on a cluster of seven dual Pentium III workstations.

Two special issues should be noted in order to simulate the SPP resonance appropriately. While the conditions near the resonance, strong evanescent waves would be built up around the surfaces of the thin film, and the electromagnetic wave propagated inside the thin film at an extremely slow group velocity, which results from the fact that the propagation constant $k_{s p}$ becomes very large in the SPP resonant condition. Because the usual PML can only absorb propagating waves, there will be serious artificial reflection if the evanescent waves encounter the boundaries. To solve the evanescent wave problem, a modified PML boundary condition was used to absorb the evanescent waves as well as the propagating waves at the boundary. ${ }^{20}$ For the slow group velocity problem, long incident pulse duration and extra-long calculation time were used for the system to have sufficient time to interact.

\section{RESULTS AND DISCUSSION}

\section{A. SPP and LSP resonance}

Figure 2 shows the calculated reflection coefficient and transmission coefficient as a function of the period for silver films with different thicknesses. There are two valleys of the reflection coefficients for the film of $116 \mathrm{~nm}$ thickness. The very sharp one is around $l=616 \mathrm{~nm}$, and the quite broad one is near $l=566 \mathrm{~nm}$. When the thickness of film decreases, the position of the sharp valley shifts slightly to longer periods, 


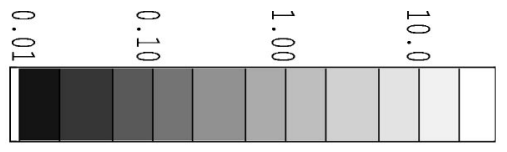

Amplitude
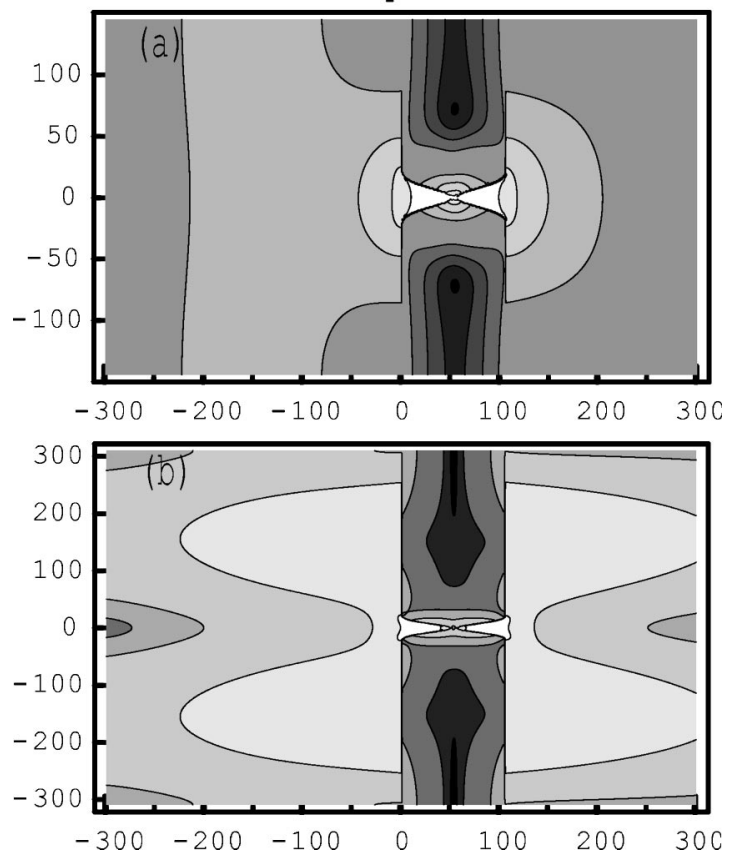

FIG. 3. Near-field distributions of periodic grooves. (a) illustrates the LSP resonance mode for $t=106 \mathrm{~nm}$ and $l=291 \mathrm{~nm}$ which is the broad resonant peak of the transmittance curve. (b) illustrates the SPP resonance mode for $t=106 \mathrm{~nm}$ and $l$ $=621 \mathrm{~nm}$ which is the narrow resonant peak of the transmittance curve. The field amplitude has been normalized for the incident field amplitude to be 1 . The length unit of $x$ and $y$ axes is in $\mathrm{nm}$.

but the broad valley moves significantly to shorter periods. Figure 2(b) shows that a peak in a transmission curve always corresponds to a minimum in the reflection curve at the same period. The transmittance peaks around $l=616-623 \mathrm{~nm}$ never exceed 0.3 , but the broad transmittance peaks can be quite large. At $t=104 \mathrm{~nm}$, the transmittance almost reaches to unity. The clear contrasts in the resonance broadness and shift suggest that they have different physical mechanisms.

The near-field distributions of these two different resonant modes in Fig. 3 are obviously different. From the dispersion relation of SPP,

$$
k_{s p}^{2}=\frac{\omega^{2}}{c^{2}} \frac{\epsilon_{\text {silver }}}{\epsilon_{\text {silver }}+1},
$$

the period to match with the real part of $k_{s p}$ is $l=617 \mathrm{~nm}$. The narrow transmission peaks shown in Fig. 2(b) can be identified as the SPP resonances, and the small deviations may result from the small thickness of the film and the grooves. By solving the dispersion relations exactly for a thin metal film surrounded by dielectric media, the real part of the propagation constant becomes smaller (in the symmetric mode) with decreasing film thickness, ${ }^{21}$ i.e., the period needed to match the in-plane momentum would be longer.
This trend was observed in our simulations. While the thickness of the films is reduced from $t=116 \mathrm{~nm}$ to $t=104 \mathrm{~nm}$, the sharp peak positions shift slightly from 616 to $623 \mathrm{~nm}$. Figure 3(b) shows a typical near-field distribution at the SPP resonance. There are strong fields on both sides of the surfaces of the film and the maxima of the fields located at $1 / 4$ and $3 / 4$ period. The near-field distribution agrees with physical pictures of the surface plasmon polariton in a periodic structure.

Similar to the field distributions analyzed in previous work, ${ }^{16,22}$ in Fig. 3(a) there are LSP modes strongly localized in the grooves on both sides of the film surfaces. Resonant excitation of a LSP mode in the left surface grooves results in strong field enhancement. This in turn resonantly excites the LSP mode on the right surface and builds a strong electromagnetic field, which finally emits the radiation downwards into the air. ${ }^{16}$ This transmission process is effectively tunneling light through a metal film via resonantly excited LSP modes.

The existence of two kinds of electromagnetic resonances in one-dimensional (1D) periodically nanostructured metals and their relation with enhanced optical transmission was reported by Porto et al. ${ }^{12}$ and they used a metallic grating with very narrow slits to demonstrate the properties of the coupled SPP resonance and the waveguide mode resonance. It is instructive to compare the surface plasmon modes from a grating with very narrow slits and those from a grating with very narrow grooves. It is clear that the same SPP resonance exists in both cases. On the other hand, the waveguide mode resonance of the grating with narrow slits cannot be supported in the model of this work. The waveguide mode resonance and the LSP resonance in this work have many similarities: both are highly localized in the slits or the grooves and both have broad transmittance peaks. They also show different characteristics. The peaks of the waveguide modes move away from the peaks of the SPP resonances and become broader with increasing grating height, the peaks of the LSP resonance move away from the peaks of the SPP resonance and become broader with decreasing grating height (film thickness). The peak transmittance coefficients of the waveguide modes are not very sensitive to the grating height, on the contrary, the peak transmittance coefficients of the LSP resonance strongly depend on the grating height (film thickness) as well as the distance between the tips of the opposite grooves. The different characteristics of the waveguide modes and the LSP modes may be suitable to different demands from various applications.

\section{B. Cavity energy and phase lag}

To study how the energy of the incident light couples into the SPP and LSP modes, we calculated the electromagnetic field energy in the grooves at the left (incident) and right (outgoing) sides of the film. For example, in the case of the film thickness $t=106 \mathrm{~nm}$, the field energy as a function of periods has two peaks correspond with the LSP and SPP resonance modes, respectively (see Fig. 4). The field energy is strongly built up in the grooves at both the LSP and the SPP resonance peaks, but the field energy in the LSP reso- 


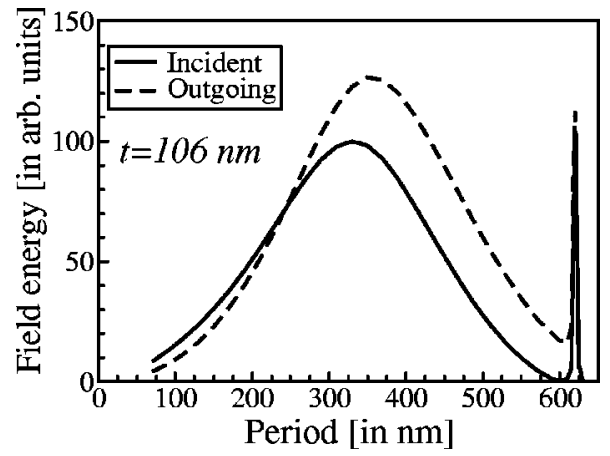

FIG. 4. Electromagnetic field energy (in arbitrary unit) in the grooves on both sides for thickness $t=106 \mathrm{~nm}$.

nance is larger than that in the SPP resonance. The field energy peaks are not at exactly the same period as the transmittance peaks. For the LSP resonance, the field energy peak of the left (incident) groove shifts to the longer period, and that of the right (outgoing) groove shifts even more at the same direction. Another interesting feature is that, at shorter periods, field energy in the left (incident) groove is larger than that in the right (outgoing) groove. On the other hand, when the periods are longer than the LSP resonance peak, the field energy in the right (outgoing) groove is larger than that in the left (incident) groove. The period of the LSP resonance peak is quite close to the position where the field energy of the left (incident) and right (outgoing) groove are the same. This behavior could be related to the coupling process of the LSP resonant modes between the grooves at both sides of the film. The SPP resonance does not exhibit such behavior.

An important feature of the general resonance phenomenon is the phase lag and the time delay in the resonant response. Dogariu et al. ${ }^{23}$ have measured the time delay of a pulse going through the silver film with a subwavelength hole array. At $800 \mathrm{~nm}$ resonance, the measured group velocity in the film can be as low as $c / 7$. It indicates that the effective index of refraction of the thin film is about 7. Their experiment agrees with a resonantly driven oscillator model and supports that the resonant coupling of light with SPP modes results in the high optical transmission and the slow group velocity of light passing through the silver film with subwavelength hole arrays. To examine the resonant responses in our simulations, we calculated the phase lag of the outgoing plane wave, comparing with the case without the silver film. The phase lag for a continuous incident light directly corresponds with the time delay for the pulsed incident light. In Fig. 5, there is no obvious phase-lag peak associated with the LSP resonance, except a smooth rising in the phase lag near the LSP resonance position of each thin film with different thickness. On the contrary, the SPP resonance exhibits large phase lag. The relation

$$
\Delta \psi=2 \pi\left(n_{e f f}-1\right) l / \lambda
$$

can be applied to obtain the effective index of refraction. At the SPP resonant peak, the effective index of refraction can reach as large as 6.9. Though the experiment by Dogariu et al. was on a two-dimensional hole array around $800 \mathrm{~nm}$ wavelength and our calculation was on a one-dimensional


FIG. 5. (a) Relative phases and (b) effective indexes of refraction as a function of period for film thickness $t=104-116 \mathrm{~nm}$.

groove array at $650 \mathrm{~nm}$ wavelength, both exhibited large effective index of refraction for the SPP resonance.

From the theory of resonantly driven oscillation, the phase lag and time delay are inversely proportional to the damping constant of the oscillator and the damping constant is proportional to the width of the resonance peak. The large phase lag of the SPP resonance accompanies the narrow SPP resonant peak. This feature infers that at the SPP resonance the damping constant is small and the $Q$ value of cavity is high. On the other hand, the insignificant phase lag and time delay in the LSP resonance is associated with the very broad LSP resonance, which suggests the damping constant of the LSP resonance is large.

\section{Implications on randomly distributed grooves}

It should be noticed that since the LSP modes are highly localized in the grooves, their response curves form very broad bands. The responses of the LSP resonance are not sensitive to the period over a large range of variation. Consequently the broad nature of the LSP resonance can be applied to the situations other than the periodic array of grooves. If the distance between the grooves are slightly different from the resonant period, we can expect the system to still have high transmittance. Therefore if the groove distances have some small random deviation from the resonant period, we still can anticipate that the system has high transmittance. This property not only gives large tolerance for future mass production of micro- or nanophotonic devices, but also provides insight on the optical properties of randomly distributed nanostructures.

To illustrate our hypothesis, we calculated the transmittance coefficients of a thin film with such randomly distrib- 
uted grooves. From the Fig. 2, the LSP resonant period $l$ is about $290 \mathrm{~nm}$ for the thin film of thickness $t=106 \mathrm{~nm}$ and the peak transmittance coefficient is about 0.69. For this case, we add random deviations in the groove positions from the resonant period. The center positions of the grooves at both sides are now $n l+v *[2 * \operatorname{random}()-1], n=\cdots,-2$, $-1,0,1,2, \ldots$, where random() is a random number from a uniformly random number generator of region $(0,1)$ and $v$ is the range of the random deviations. For $v=29 \mathrm{~nm}(10 \%$ of the resonant period $l$ ) the average transmittance coefficient is $0.648 \pm 0.027$ and for $v=58 \mathrm{~nm}(20 \%$ of the resonant period $l$ ) the average transmittance coefficient is $0.579 \pm 0.019$. They are only 94 and $84 \%$ of the peak transmittance at the resonant period respectively, and these examples confirm our hypothesis.

Another example is the super-resolution near-field structure (super-RENS), ${ }^{24}$ which exhibits dynamical nonlinear behaviors ${ }^{25-27}$ and strong local near-fields, ${ }^{27-29}$ and has vast potential on applications like high-density optical storage systems and photonic transistors. ${ }^{30}$ The Sb-type super-RENS has very rough interfaces between the 15-nm Sb layer and the protective dielectric layers. ${ }^{29}$ The rough interfaces look like randomly distributed narrow grooves and may allow strong LSP resonance when the super-RENS was excited with incident light. This hypothesis can explain the strong local near field and unusual high transmittance of the superRENS. The very broad nature of the LSP resonance can also explain why the super-RENS responses effectively to different frequencies of incident light. The physical mechanism of another $\mathrm{AgO}_{x}$-type super-RENS may also be explained in a similar way. The only difference is that the LSP resonance is not at small narrow grooves, but at the randomly distributed, nanosize silver particles embedded in the $\mathrm{AgO}_{x}$ layer. ${ }^{29}$

\section{CONCLUSION}

In conclusion, we have demonstrated different mechanisms for strong optical transmission through metallic films. By modeling a silver film, which has an array of periodic narrow grooves on both surfaces, we have shown that, via resonantly exciting the SPP and LSP modes, TM-polarized light may tunnel through the film (with a transmittance close to 1 at LSP resonance), while TE-polarized light will be almost completely reflected by the film. Though the electromagnetic field energy of cavities was built up at both LSP and SPP resonant conditions, different resonance modes have distinguishable features. SPP mode has very narrow resonance peak, which corresponds to large phase lag and time delay. Our model presents high effective index of refraction for the SPP resonance and this feature was also demonstrated by the previous experiment on a two-dimensional hole array. LSP modes have broad resonance, and their near-field distributions are highly concentrated in the grooves at both sides of the film. The special characteristics of the LSP modes may extend our understanding on the optical properties of randomly distributed metallic structures.

Our results not only bring insight on the different physical mechanisms of the unexpected strong light transmission through metal films with subwavelength hole arrays or groove arrays, but they may also lead to micro- and nanophotonics applications. Since the LSP resonance is closely dependent on the details of nanoscale structures, it is hard to predict what kind of LSP resonance modes would appear in a two-dimensional, periodic metallic structure. Nevertheless, similar local-field excitation phenomena have often been observed in the near field optical research, and it is reasonable to expect the LSP resonance in various metallic nanostructures from further experiments. Up to now, little is known about the optical near fields around nanoscale metal structures, nor about the LSP modes excited by incident light. Better understanding of such knowledge is greatly desirable in order to manipulate and localize light in subwavelength scale.

\section{ACKNOWLEDGMENTS}

The authors are grateful for the research support by the National Science Council, Taiwan, R.O.C., and the Ritek Corporation.
*Electronic address: wcliu@phy.ntnu.edu.tw

${ }^{1}$ V.M. Agranovich and D.L. Mills, Surface Polaritons: Electromagnetic Waves at Surfaces and Interfaces (North-Holland, Amsterdam, 1982).

${ }^{2}$ H. Raether, Surface Plasmons (Springer, New York, 1988).

${ }^{3}$ T.W. Ebbesen, H.J. Lezec, H.F. Ghaemi, T. Thio, and P.A. Wolff, Nature (London) 391, 667 (1998).

${ }^{4}$ T. Thio, H.F. Ghaemi, H.J. Lezec, P.A. Wolff, and T.W. Ebbesen, J. Opt. Soc. Am. B 16, 1743 (1999).

${ }^{5}$ T. Thio, H.J. Lezec, and T.W. Ebbesen, Physica B 279, 90 (2000).

${ }^{6}$ D.E. Grupp, H.J. Lezec, T.W. Ebbesen, K.M. Pellerin, and T. Thio, Appl. Phys. Lett. 77, 1569 (2000).

${ }^{7}$ H.A. Bethe, Phys. Rev. 66, 163 (1944).

${ }^{8}$ I. Avrutsky, Y. Zhao, and V. Kochergin, Opt. Lett. 25, 595 (2000).

${ }^{9}$ E. Popov, M. Nevière, S. Enoch, and R. Reinisch, Phys. Rev. B 62, 16100 (2000).

${ }^{10}$ L. Salomon, F. Grillot, A.V. Zayats, and F. de Fornel, Phys. Rev.
Lett. 86, 1110 (2001).

${ }^{11}$ L. Martín-Moreno, F.J. García-Vidal, H.J. Lezec, K.M. Pellerin, T. Thio, J.B. Pendry, and T.W. Ebbesen, Phys. Rev. Lett. 86, 1114 (2001).

${ }^{12}$ J.A. Porto, F.J. García-Vidal, and J.B. Pendry, Phys. Rev. Lett. 83, 2845 (1999).

${ }^{13}$ U. Schröter and D. Heitmann, Phys. Rev. B 58, 15419 (1999).

${ }^{14}$ S. Astilean, P. Lalanne, and M. Palamaru, Opt. Commun. 175, 265 (2000).

${ }^{15}$ Y. Takakura, Phys. Rev. Lett. 86, 5601 (2001).

${ }^{16}$ W.-C. Tan, T.W. Preist, and J.R. Sambles, Phys. Rev. B 62, 11134 (2000).

${ }^{17}$ A. Taflove, Computational Electrodynamics: The FiniteDifference Time-Domain Method (Artech House, Boston, 1995).

${ }^{18}$ J.B. Judkins and R.W. Ziolkowski, J. Opt. Soc. Am. A 12, 1974 (1995).

${ }^{19}$ S.D. Gedney, IEEE Trans. Antennas Propag. 44, 1630 (1996). 
${ }^{20}$ S.D. Gedney, in Advances in Computational Electrodynamics: The Finite-Difference Time-Domain Method, edited by A. Taflove (Artech House, Boston, 1998) Chap. 5, p. 263.

${ }^{21}$ J.J. Burke, G.I. Stegeman, and T. Tamir, Phys. Rev. B 33, 5186 (1986).

${ }^{22}$ W.-C. Tan, T.W. Preist, R.J. Sambles, and N.P. Wanstall, Phys. Rev. B 59, 12661 (1999).

${ }^{23}$ A. Dogariu, T. Thio, L.J. Wang, T.W. Ebbesen, and H.J. Lezec, Opt. Lett. 26, 450 (2001).

${ }^{24}$ J. Tominaga, T. Nakano, and N. Atoda, Appl. Phys. Lett. 73, 2078 (1998).

${ }^{25}$ T. Fukara, J. Tominaga, T. Nakano, and N. Atoda, Appl. Phys.
Lett. 75, 3114 (1999).

${ }^{26}$ J. Tominaga, H. Fuji, A. Sato, T. Nakano, and N. Atoda, Jpn. J. Appl. Phys., Part 1 39, 957 (2000).

${ }^{27}$ H. Fukaya, D. Buechel, S. Shinbori, J. Tominaga, N. Atoda, D.P. Tsai, and W.C. Lin, J. Appl. Phys. 89, 6139 (2001).

${ }^{28}$ D.P. Tsai and W.C. Lin, Appl. Phys. Lett. 77, 1413 (2000).

${ }^{29}$ W.-C. Liu, C.-Y. Wen, K.-H. Chen, W.C. Lin, and D.P. Tsai, Appl. Phys. Lett. 78, 685 (2001).

${ }^{30}$ J. Tominaga, C. Mihalcea, D. Buechel, H. Fukuda, T. Nakano, N. Atoda, H. Fuji, and T. Kikukawa, Appl. Phys. Lett. 78, 2417 (2001). 